\title{
REVISIÓN SOBRE LA AGUDEZA VISUAL DINÁMICA
}

\section{Review of dynamic visual acuity}

\author{
Pablo SANTOS-GORJÓN \\ SACYL. Complejo Hospitalario de Ávila. Servicio de Otorrinolaringología. Ávila. España \\ Correspondencia: pablosantosgor@gmail.com
}

Fecha de recepción: 23 de octubre de 2017

Fecha de aceptación: 6 de diciembre de 2017

Fecha de publicación: 8 de diciembre de 2017

Fecha de publicación del fascículo: 1 de junio de 2018

Conflicto de intereses: Los autores declaran no tener conflictos de intereses

Imágenes: Los autores declaran haber obtenido las imágenes con el permiso de los pacientes Política de derechos y autoarchivo: se permite el autoarchivo de la versión post-print (SHERPA/RoMEO) Licencia CC BY-NC-ND. Licencia Creative Commons Atribución-NoComercial-SinDerivar 4.0 Internacional Universidad de Salamanca. Su comercialización está sujeta al permiso del editor

RESUMEN

PALABRAS CLAVE

SUMMARY

Introducción y objetivo: Para mantener la mirada estable durante los rápidos movimientos de cabeza que realizamos en nuestra vida diaria, es necesario que el sistema vestibular detecte la velocidad cefálica y a través del reflejo vestíbulo-ocular (RVO) induzca un movimiento del ojo de igual magnitud y de dirección opuesta. Si este mecanismo falla, como ocurre con la hipofunción vestibular, el paciente se queja de visión borrosa (oscilopsia). Una manera de comprobar el funcionamiento de este reflejo es mediante la cuantificación de la agudeza visual dinámica (AVD). En este trabajo describiremos las pruebas que permiten su valoración. Material y métodos: Revisión narrativa. Resultados: Hay dos tipos de herramientas que nos permiten medir la agudeza visual dinámica (AVD), las pruebas clínicas y las computarizadas. Se puede aumentar la fiabilidad de la prueba clínica, si en la evaluación añadimos algún sistema que nos ayude a controlar la frecuencia y la amplitud del movimiento que inducimos a la cabeza. Discusión: Las pruebas clínicas son más sencillas, accesibles y económicas y, sin embargo, las computarizadas son más precisas, pero requieren de un instrumental más caro y menos cercano. Conclusión: Aun disponiendo para la exploración vestibular de una amplia variedad de procedimientos objetivos, la determinación clínica de la AVD sigue resultando útil para cribar la función vestibular y para medir el grado de compensación, durante la rehabilitación.

agudeza visual dinámica; pruebas vestibulares; reflejo vestíbulo-ocular; hipofunción vestibular; rehabilitación vestibular.

Introduction: To maintain gaze on a stationary target despite rapid cephalic movements we make in our everyday life, it is necessary that the vestibular system detects the velocity of the head and through the vestibular-ocular reflex (VOR) induce a movement of the eye of equal magnitude and opposite direction. If this mechanism fails, as happens with the vestibular hypofunction, the patient complains of blurred vision (oscillopsia). One way of checking the operation of this reflex is through quantification of visual acuity during head movements. In this paper, we will describe tests that allow its valuation. Method: Narrative review. Results: There are two

Ediciones Universidad de Salamanca / CC BY-NC-ND [121]

Rev. ORL, 2018, 9, 2, pp. 121-126 
types of tools that allow us to measure the dynamic visual acuity (DVA), the clinical tests, and the computerized. Reliability can be increased if in the clinical evaluation we add some system that allows us to control the frequency and the amplitude of the movement which we induce to the head. Discussion: Clinical tests are more simple, accessible and cost-effective, and however, the computerized are more accurate, but require more expensive and less close instruments. Conclusion: Even featuring a variety of objective procedures, the clinical determination of the DVA remains useful for screening the vestibular function and to measure the degree of compensation, during rehabilitation.

KEYWORDS

dynamic visual acuity; vestibular testing; vestibule-ocular reflex; vestibular hypofunction; vestibular rehabilitation.

\section{INTRODUCCIÓN}

La agudeza visual es la capacidad para percibir, detectar e identificar con la vista, determinados objetos. A una misma distancia, si un paciente ve nítidamente una letra, tiene más agudeza visual que otro que no la ve. La agudeza visual estática (AVE) es la capacidad de reconocer objetos en reposo y la agudeza visual dinámica (AVD) mide la discriminación visual cuando el observador o el objeto observado se mueven. Esta competencia resulta fundamental en algunas actividades como la conducción o la práctica deportiva, entre otras. Así, se ha objetivado que la AVD es superior en los jugadores de waterpolo que, en una muestra de estudiantes sedentarios con similar agudeza visual estática, sugiriendo que el entrenamiento de esta capacidad visual es efectivo, pudiendo incluso ser un buen predictor del éxito en determinadas modalidades deportivas [1, 2]. El término AVD fue acuñado en 1949 por Ludvigh y Miller [3], pero la primera publicación que utiliza la AVD en pacientes con alteración vestibular fue la de Longridge y Mallinson en 1984 [4]. Se define como el umbral de resolución visual obtenido durante el movimiento cefálico del sujeto o del estímulo visual, que suele tratarse de optotipos (símbolos estandarizados utilizados para las pruebas de visión) [5]. En condiciones normales, la agudeza visual disminuye en función de la velocidad de movimiento de los optotipos según una relación matemática precisa, directamente relacionada con el deterioro del seguimiento lento [6]. Cuando es el sujeto quien se mueve, entra en acción el reflejo vestíbulo ocular (RVO), mejorando la calidad de la señal [5].

Dado que la AVD puede medirse clínicamente, con este trabajo pretendemos presentar las distintas pruebas que permiten dicha medición y el significado de los resultados obtenidos.

FISIOPATOLOGÍA

Cuando giramos la cabeza en una dirección, para que la imagen del objeto se mantenga estable en la retina, el ojo debe moverse en dirección contraria, con la misma velocidad (ganancia) y en el mismo momento (fase) que la cabeza [7]. Si el movimiento cefálico es lento $(<1 \mathrm{~Hz})$, el desplazamiento de la imagen sobre la retina se compensa mediante la activación de los sistemas de seguimiento visual y optoquinético, evitando de esta forma que el desplazamiento retiniano sobrepase ciertos límites [5]. Pero la mayoría de los movimientos que realizamos en la vida diaria son de alta frecuencia, pudiendo alcanzar $5-10 \mathrm{~Hz}$ [6] y en estos casos este mecanismo resulta ineficaz, requiriéndose la participación del reflejo vestíbulo-ocular (RVO) [7]. El RVO conecta neuralmente el aparato vestibular con los músculos oculomotores. Su desarrollo evolutivo proviene de la necesidad de estabilizar la imagen en la retina durante los movimientos rápidos de la cabeza (caminar, correr), de manera que la interacción entre la información visual y vestibular logra una imagen macular más precisa que la obtenida solo por el sistema ocular [8]. Al mirar una diana a una distancia superior a $1 \mathrm{me}-$ tro, si funciona normalmente el RVO angular, la estabilidad de la mirada asegura una visión clara durante el movimiento de la cabeza [9]. Las características del reflejo son modificadas por las condiciones del objeto que se ha de fijar, por el ambiente visual y por las propiedades refractarias del ojo del individuo [5].

Uno de los síntomas frecuentemente referidos por los pacientes con enfermedad vestibular es una sensación de borrosidad visual con los movimientos de cabeza. A esta sensación la denominamos oscilopsia [10] y constituye un problema importante porque contribuye a la evitación de algunas actividades como la conducción, limitando incluso la interacción social que favorece el aislamiento [11]. La oscilopsia ocurre cuando la ganancia del RVO es inadecuada y sugiere una falta de compensación de la pérdida vestibular. La medida de la agudeza visual durante los movimientos de la cabeza ofrece al clínico un procedimiento útil para 
valorar el impacto de la pérdida de función vestibular, así como un medio para determinar la efectividad de aquellas intervenciones que promuevan la compensación. Dos herramientas se han desarrollado para cuantificar la agudeza visual durante el movimiento de la cabeza, las pruebas clínicas y las computarizadas [11].

\section{VALORACIÓN DE LA AGUDEZA VISUAL DINÁMICA \\ PRUEBAS CLÍNICAS}

Las pruebas de AVD, en las que o bien el paciente mueve su cabeza (test activo) o es el clínico quien se la mueve (test pasivo) mientras éste lee una carta de agudeza visual, se han utilizado en exploración vestibular desde principios de los años 80 del siglo $X X[4,8,12]$. La realización pasiva de la exploración, guiando la cabeza el examinador, tiene la ventaja de eliminar movimientos lentos preprogramados compensatorios [5]. Nos colocamos por detrás del paciente y le explicamos que deje el cuello relajado. Debemos poder mover la cabeza a 1,5 a $2 \mathrm{~Hz}$ de frecuencia con movimientos horizontales de poca amplitud, sin sobrepasar los 30 a 40 grados [13].

El paciente se sienta a unos $3 \mathrm{~m}$ del objetivo visual, para evitar los efectos de la acomodación, que a esta distancia son despreciables [8]. La prueba clínica más habitual utiliza como diana la tabla de Snellen, diseñada en 1862 para evaluar la agudeza visual y que consiste en un conjunto de diez letras - B, C, D, E, F, L, $\mathrm{O}, \mathrm{P}, \mathrm{T}$ y la $\mathrm{Z}$-, con un tamaño decreciente dependiendo del nivel en el que se encuentren. Un nivel 20/20 corresponde a una visión normal. Sin embargo, se discute la conveniencia de utilizar esta tabla, porque las letras no son igualmente legibles a pesar de tener el mismo tamaño y porque dado el escaso número de letras, la persona a examinar podría aprenderlas de memoria [8]. En los primeros estudios clínicos sobre la AVD, Longridge y Mallinson $[4,8]$ desarrollaron la prueba dinámica de la $\mathrm{E}$ ilegible o DIE test, que consiste en una serie de filas, donde se repite la letra $E$ mayúscula en diferentes posiciones (abierta hacia arriba, hacia abajo, a la derecha y a la izquierda).

Con la cabeza estacionaria delante de una tabla de Snellen, preguntamos al paciente qué letras ve, y le pedimos que vaya identificando los caracteres de derecha a izquierda, descendiendo hacia las filas con letras más pequeñas (lo mismo en el DIE test, indicando hacia qué lado está abierta cada letra E). La última línea para considerar es aquella en la que el sujeto discrimina al menos, el $50 \%$ de los optotipos [11] y marca su agudeza visual estática (AVE). El paciente debe ser capaz de leer las 4 primeras filas y si no lo es, se le aproxima a la mitad de distancia. Si aún no puede leerlas, se le excluye de la prueba [8]. A continuación, medimos el cambio de valor de la agudeza visual durante la realización de movimientos cefálicos horizontales y verticales (AVD). Los resultados se cuantifican determinando la diferencia entre la línea de optotipos más pequeños que es capaz de leer con la cabeza estacionaria y la que es capaz de leer con la cabeza en movimiento. Es normal que se produzca un cierto deterioro visual durante el movimiento, perdiéndose una línea, teniendo que acudir a la superior en tamaño. Por tal motivo, la mayoría de los clínicos aplica un cambio de dos o más líneas como indicador de patología [11]. Gráficas similares son descargables de internet, pudiendo utilizar otras con diferentes caracteres a la estándar, a fin de evitar la memorización en caso de repetición al mismo paciente.

Al realizar la prueba, es fundamental utilizar siempre las mismas condiciones, respecto a la distancia de lectura, luminosidad y corrección de ametropía. Si el paciente utiliza lentes (de contacto o gafas), la exploración se realiza con ellas, excepto en los casos de lentes progresivas donde la prueba se desarrolla sin corrección [11].

Otra opción, es presentar un solo optotipo (letra o número) durante un segundo, mientras se mueve la cabeza del sujeto, apuntándose las respuestas correctas en vez del número de líneas leídas [14].

Finalmente, una tercera alternativa consiste en presentar las dianas en un ordenador, utilizando números de tamaño determinado. Los cambios de tamaño son similares a los de la carta de Snellen, de línea a línea. El paciente tiene 3 segundos para identificar una serie de 5 números, todos del mismo tamaño [15].

En nuestro medio, Oliva et al. [7] han publicado el test de lectura, como método de valoración cualitativa del RVO. Se utiliza un texto con un tamaño y tipo de letra legible (Times New Roman, 12 puntos) y con un contenido de vocabulario sencillo. El paciente coge el texto con las manos y comienza a leerlo en voz alta. Situados por detrás, se le comienza a mover la cabeza incrementando la velocidad, hasta alcanzar una frecuencia de 2 a $3 \mathrm{~Hz}$. Si la 
estabilidad visual es deficiente, el paciente no podrá continuar leyendo [16]. Se trata de una prueba sencilla, cómoda, rápida, económica y fácil de valorar, que podría servir para el cribado de la función vestibular periférica [7].

\section{PRUEBAS COMPUTARIZADAS}

Además de las pruebas clínicas descritas, la AVD puede analizarse mediante procedimientos computarizados [11]. En este caso, la diana sólo se muestra cuando la velocidad de la cabeza o su rango frecuencial están dentro de unos parámetros predeterminados. El equipo consta de un sensor que mide el movimiento de la cabeza y en general, el umbral de velocidad que permite la aparición del optotipo está por encima de los $120 \%$ s, porque a esta velocidad el seguimiento lento no contribuye a la estabilidad de la mirada. El programa presenta aleatoriamente los optotipos, cuyo tamaño es el exacto para un descenso determinado de agudeza visual. Finalmente, permite diferenciar la agudeza visual en ambos lados, hacia el lado sano y hacia el que presente una supuesta hipofunción vestibular [17].

Se puede hacer una valoración de la AVD también en niños, con objetos de su interés, para que la exploración sea entretenida y no agresiva [18]. En personas que no saben leer, tienen problemas de dislexia o en sus idiomas no se usan caracteres latinos, además de la carta de Snellen hay otros métodos de evaluación, como la prueba DIE $[4,8]$ o el test de Landolt con anillos discontinuos donde el paciente debe discernir hacia qué lado está abierto [19]. En ocasiones, además de medir la AVD en el plano horizontal se hace también en el vertical, así por ejemplo tras la resección de un neurinoma del VIII par, los pacientes manifiestan una alteración de la AVD tanto en los movimientos horizontales, como en los verticales, dado que en la cirugía se seccionan las aferencias del canal semicircular horizontal, y las de los canales verticales [13].

No disponemos de un examen que proporcione una medida de la AVD en diferentes contextos de la vida cotidiana, tales como los propios de la práctica deportiva o del ámbito de la conducción de vehículos, que permita un análisis de los procesos básicos de la percepción del movimiento, tan importantes para la seguridad viaria o para establecer comparaciones con grupos de riesgo (cataratas, glaucoma, retinopatías, baja visión, etc.). Sí sabemos, por los estudios realizados, que no parece que existan diferencias estadísticamente significativas ligadas al género [20].

Además, la AVD puede resultar de utilidad para evaluar la eficacia clínica de un tratamiento rehabilitador, comparando los resultados obtenidos antes y después del mismo [11]. Mediante los ejercicios de estabilización de la mirada, que buscan mover las imágenes visuales fuera de la fóvea de la retina, para provocar un "deslizamiento retiniano", sabemos que se mejora la AVD en pacientes con hipofunción vestibular uni y bilateral $[9,17,21]$.

\section{DISCUSIÓN}

Como con cualquier evaluación, la medida de la AVD debe demostrar suficiente precisión y validez en la población de interés, a través de las características que la definan. Estas son, la velocidad, la frecuencia y el plano del movimiento de cabeza, en rangos que reflejen la contribución vestibular a la estabilidad de la mirada. Sin evidencia de una moderada precisión, el clínico no puede asegurar que los cambios en el resultado expresen una mejoría o sean fruto de la casualidad [11].

Aunque las pruebas clínicas son las idóneas, porque son económicas, requieren poco equipamiento y pueden ser realizadas en la propia consulta [13], tienen problemas como instrumento de medida. Su precisión entre sujetos y en el mismo sujeto, es escasa en pacientes con hipofunción vestibular [11] y muestran una importante variabilidad en la amplitud, velocidad y frecuencia de movimiento de la cabeza durante cada prueba. El uso de un metrónomo para estandarizar la frecuencia y un preciso control de la amplitud del movimiento pueden mejorar su precisión [13, 22]. Generalmente se sigue el compás de una señal auditiva a $2 \mathrm{~Hz}$ y se controla la amplitud del movimiento en $40^{\circ}$, lo que da como resultado una velocidad pico de la cabeza de 160\%/s, pero la precisión y validez no están absolutamente establecidas $[4,8,13]$. Por su parte, en los servicios de otorrinolaringología no se suele disponer de equipos computarizados [13].

La AVD está afectada en pacientes con insuficiencia vestibular bilateral y se detecta tanto con procedimientos computarizados como no computarizados [23]. Sin embargo, en el diagnóstico de la hipofunción vestibular unilateral, aunque las pruebas computarizadas han demostrado su sensibilidad y especificidad [11], resulta más complejo conocer la efectividad de las pruebas clínicas [13, 23]. Los pacientes con 
déficit vestibular unilateral y afectación de la prueba de AVD, no suelen quejarse de oscilopsia en su vida diaria [17, 24]. Esto puede explicarse porque se adoptan estrategias de comportamiento que evitan los movimientos rápidos hacia el lado lesionado. Además, durante los giros hacia dicho lado, se produce un desplazamiento de los ojos, anticipatorio al movimiento de la cabeza [23]. Sin embargo, muchos de estos pacientes sí experimentan oscilopsia durante la prueba de AVD, al moverles rápidamente la cabeza, sugiriendo que la condición de esta prueba es diferente a lo acontecido en la vida diaria, probablemente por el uso de los mecanismos compensatorios explicados. Algunos autores encuentran anomalías en las pruebas clínicas de AVD en la mayoría de los pacientes con una hipofunción vestibular unilateral, cuando la frecuencia del movimiento de cabeza es $\geq 1,5 \mathrm{~Hz}[13,23]$. Sin embargo, la prueba de AVD no permite distinguir diferentes grados de afectación, como lo hace la prueba calórica, que por otra parte sólo evalúa a baja frecuencia los conductos horizontales [11, 13, 23], mientras que, como hemos dicho, la AVD también permite el análisis de los verticales.

Un problema añadido de las pruebas clínicas es la falta de unificación de criterios en el procedimiento administrado, donde el rango, la dirección y la frecuencia de oscilación de la cabeza varía de unas a otras publicaciones [13]. Parece comprobado que la mejor frecuencia de evaluación clínica de la AVD, tanto para movimientos horizontales como verticales es la de $1,5 \mathrm{~Hz}[4,8,13]$, disminuyendo los falsos positivos (prueba patológica en sujetos sanos). También parece que la prueba DIE [4, 8] produce resultados más consistentes que la de la carta de Snellen $[4,8,13]$.

Los pacientes con una miopía importante son incapaces de leer bien con la cabeza en movimiento y se debe tener en cuenta que si los órganos vestibulares periféricos funcionan adecuadamente (comprobado mediante la prueba térmica), una AVD patológica implicaría una alteración a nivel central (tronco del encéfalo o cerebelo) en alguna región de la vía del RVO [8].

\section{CONCLUSIONES}

En el contexto de la exploración vestibular, la evaluación clínica de la AVD es un procedimiento rápido y poco molesto que se sitúa entre los procedimientos objetivos clásicos (prueba térmica, sillón rotatorio, VEMPs) y la observación clínica subjetiva [25]. Es un indicador del funcionamiento del RVO y se encuentra afectado en pacientes con pérdida bilateral de la función vestibular y en algunos con hipofunción unilateral. Los cambios observados en la AVD a lo largo del tiempo pueden indicar el desarrollo de mecanismos de compensación.

Por tanto, las pruebas clínicas que estudian la AVD resultan de utilidad para cribar la función vestibular y como indicadores de compensación, durante la rehabilitación.

\section{BIBLIOGRAFÍA}

1. Hillier SL, Hollohan V. Vestibular rehabilitation for unilateral peripheral vestibular dysfunction. Cochrane Database Syst Rev. 2007;(4):CD005397. Review. Update in: Cochrane Database Syst Rev. 2011;(2):CD005397.

2. Mraz M, Curzytek M, Mraz MA, Gawron W, Czerwosz L, Skolimowski T. Body balance in patients with systemic vertigo after rehabilitation exercise. J Physiol Pharmacol. 2007;58 Suppl 5(Pt 1):427-36.

3. Miller JW, Ludvigh E. The effect of relative motion on visual acuity. Surv Ophthalmol. 1962;7:83-116.

4. Longridge NS, Mallinson Al. A discussion of the dynamic illegible "E" test: a new method of screening for aminoglycoside vestibulotoxicity. Otolaryngol Head Neck Surg. 1984;92(6):671-7.

5. Pérez Fernández N. Anamnesis y exploración clínica otoneurológica. En: Bartual Pastor J, Pérez Fernández N. El sistema vestibular y sus alteraciones. $1^{\text {a }}$ ed. Barcelona: MASSON, S.A.; 1998. p. 83-103.

6. Demer JL, Honrubia V, Baloh RW. Dynamic visual acuity: a test for oscillopsia and vestibulo-ocular reflex function. Am J Otol. 1994;15(3):340-7.

7. Oliva Domínguez M, Martín García M, Dañino González J, Dañino González G, Bartual Pastor J. El test de lectura como método de valoración cualitativa del reflejo vestíbulo-ocular. Acta Otorrinolaringol Esp. 1999;50(5):371-6. 
8. Longridge NS, Mallinson Al. The dynamic illegible E-test. A technique for assessing the vestibulo-ocular reflex. Acta Otolaryngol. 1987;103(3-4):273-9.

9. Schubert MC, Migliaccio AA, Clendaniel RA, Allak A, Carey JP. Mechanism of dynamic visual acuity recovery with vestibular rehabilitation. Arch Phys Med Rehabil. 2008;89(3):500-7.

10. Hess K, Gresty M, Leech J. Clinical and theoretical aspects of head movement dependent oscillopsia (HMDO). A review. J Neurol. 1978;219(3):151-7.

11. Herdman SJ. Computerized dynamic visual acuity test in the assessment of vestibular deficitis. En: Vertigo and Imbalance: Clinical Neurophysiology of the Vestibular System. Handbook of Clinical Neurophysiology, Vol 9. $1^{\underline{a}}$ ed. Elsevier; 2010. p. 181-190.

12. Burgio DL, Blakley BW, Myers SF. The high-frequency oscillopsia test. J Vestib Res. 1992 Fall;2(3):221-6.

13. Dannenbaum E, Paquet N, HakimZadeh R, Feldman AG. Optimal parameters for the clinical test of dynamic visual acuity in patients with a unilateral vestibular deficit. J Otolaryngol. 2005;34(1):13-9.

14. Grossman GE, Leigh RJ, Bruce EN, Huebner WP, Lanska DJ. Performance of the human vestibuloocular reflex during locomotion. J Neurophysiol. 1989;62(1):264-72.

15. Hillman EJ, Bloomberg JJ, McDonald PV, Cohen HS. Dynamic visual acuity while walking in normals and labyrinthine-deficient patients. J Vestib Res. 1999;9(1):49-57.

16. Oliva M. Manual de exploración vestibular para residentes de ORL y algunos adjuntos, especialmente torpes para estos menesteres. $2^{\mathrm{a}}$ ed. GAES; 34p.
17. Herdman SJ, Schubert MC, Das VE, Tusa RJ. Recovery of dynamic visual acuity in unilateral vestibular hypofunction. Arch Otolaryngol Head Neck Surg. 2003;129(8):819-24.

18. Hyvärinen L, Näsänen $R$, Laurinen $P$. New visual acuity test for pre-school children. Acta Ophthalmol (Copenh). 1980;58(4):507-11.

19. Danilova MV, Bondarko VM. Foveal contour interactions and crowding effects at the resolution limit of the visual system. J Vis. 2007;7(2):25.1-18.

20. Klavora P, Gaskovski P, Forsyth RD. Test-retest reliability of three Dynavision tasks. Percept Mot Skills. 1995;80(2):607-10.

21. Herdman SJ, Hall CD, Schubert MC, Das VE, Tusa RJ. Recovery of dynamic visual acuity in bilateral vestibular hypofunction. Arch Otolaryngol Head Neck Surg. 2007;133(4):383-9.

22. Roberts RA, Gans RE, Johnson EL, Chisolm TH. Computerized dynamic visual acuity with volitional head movement in patients with vestibular dysfunction. Ann Otol Rhinol Laryngol. 2006;115(9):658-66.

23. Dannenbaum E, Paquet N, Chilingaryan $G$, Fung J. Clinical evaluation of dynamic visual acuity in subjects with unilateral vestibular hypofunction. Otol Neurotol. 2009;30(3):368-72.

24. Schubert MC, Herdman SJ, Tusa RJ. Vertical dynamic visual acuity in normal subjects and patients with vestibular hypofunction. Otol Neurotol. 2002;23(3):372-7.

25. Peters BT, Mulavara AP, Cohen HS, Sangi-Haghpeykar $\mathrm{H}$, Bloomberg JJ. Dynamic visual acuity testing for screening patients with vestibular impairments. J Vestib Res. 2012;22(2):145-51. 